\title{
Training Data Set Assessment for Decision-Making in a Multiagent Landmine Detection Platform
}

\author{
Johana Florez-Lozano \\ Pontificia Universidad Javeriana \\ Bogota, Colombia \\ johana.florez@javeriana.edu.co
}

\author{
Fabio Caraffini \\ De Montfort University \\ Leicester, UK \\ fabio.caraffini@dmu.ac.uk
}

\author{
Carlos Parra \\ Pontificia Universidad Javeriana \\ Bogota, Colombia \\ carlos.parra@javeriana.edu.co
}

\author{
Mario Gongora \\ De Montfort University \\ Leicester, UK \\ mgongora@dmu.ac.uk
}

\begin{abstract}
Real-world problems such as landmine detection require multiple sources of information to reduce the uncertainty of decision-making. A novel approach to solve these problems includes distributed systems, as presented in this work based on hardware and software multi-agent systems. To achieve a high rate of landmine detection, we evaluate the performance of a trained system over the distribution of samples between training and validation sets. Additionally, a general explanation of the data set is provided, presenting the samples gathered by a cooperative multi-agent system developed for detecting improvised explosive devices. The results show that input samples affect the performance of the output decisions, and a decisionmaking system can be less sensitive to sensor noise with intelligent systems obtained from a diverse and suitably organised training set.
\end{abstract}

Index Terms-Land mine detection, improvised explosive device, neuroevolution, genetic fuzzy systems, decision making

\section{INTRODUCTION}

Land mines are considered the most problematic kind of unexploited ordnance (UXO) since they can remain active and dangerous for many years after being concealed. Moreover, they affect the development of communities limiting the use of land where they are concealed and in general are in detriment to health. Generally, there are two types of mines relating to their construction. Military land mines are designed under a set of specifications to target humans or vehicles as tanks, as they pass over or near them [1]. Handmade devices called improvised explosive devices (IEDs) with no recorded construction specifications, very cheap, easy to make, to deploy and more difficult to be detected with the techniques currently available in the literature (an exhaustive review is available in [2]). This means that a general scheme does not exits, and in turn, that a wide range of features must be taken into consideration to design a general detection method.

Sensing land characteristic and detecting buried objects is very complex and no one sensor or method can achieve this optimally due to several issues, such as the presence of obstacles, the variation in temperature and humidity at different times, the type of ground, among others; this limits the desired (high) detection rate [3]. Hence, the idea of investigating Artificial Intelligence (AI) based data fusion methods arose, with the aim of aggregating heterogeneous pieces of information onto a

This research received funds from De Montfort University, Colciencias [grant 647, 2014] and Pontificia Universidad Javeriana [grant VRI-05,2017]. robust distributed Decision-Making (DM) system implemented on a robotic platform.

The work proposed in this article is part of the evaluation of the performance of a cooperative and distributed decisionmaking system; whereby the distribution of tests samples among two sets, training and validation data is done such that it maximised the limited data available. The details and results of the tests performed are presented as follows:

- section II provides a brief background and context to this research by discussing limitations of the current state of the art, revising literature in land-mine detection;

- section III introduces and describes the hardware and software system which acquired the samples evaluated;

- section IV] gives details on the experimental phase and presents some results;

- section $\mathrm{V}$ draws the conclusions of this work and presents plans.

\section{CurRent Research}

Recent research in land mine detection aims at increasing accuracy in detecting mines, e.g., a machine-learning sensor fusions method is proposed in [4] to lower the false-positive rate, and moves towards the automation of risky manual work done by operators, who still may have to use dog units [5] or terrain-scanning devices [6]. In addition, there is much research around robotic platforms for searching the land for safer mine detection, more recently aiming to take the acquisition away from the hazardous terrain, e.g., the systems in [7] and [8] use a drone to scan a test terrain with a Ground Penetrating Radar (GPR).

However, there is a necessity to reach a higher degree of integration between multiple hardware and software developments, i.e., robotic platforms, sensors, decision-making algorithms and AI algorithms to produce intelligent behaviour and enhance performance. Little has been done to include multiple sensors and search explicitly for improvised explosive devices.

As part of the solution, this piece of research presents the assessment of the training and validation data distribution according to the performance of a cooperative and distributed decision-making system (CoD2M). This decision-making system executes in a decentralised and self-organising manner, called here Multi-Agent Perception System (MAPS), inspired 
by successful studies as those in [9], [10], [11], [12] to detect buried objects in the ground. The complete data set that each agent used in this study can be found in the repository [13].

\section{Multi-Agent System}

Our data set is generated by a multi-agent system described by two parts: hardware and software.

The hardware part consists of a multi-agent perception system (MAPS) with five physical agents (see Figure 11. Each agent is equipped with a specific sensor, i.e, a visual spectrum camera (VS), a near-infrared camera (IR), a nearultraviolet camera (UV), a beam thermal sensor (TM), or a light GPR (GP); each with a Single Board Computer (SBC), and a servomotor as an actuator. The sensors in the MAPS were selected from the performance and advantages of a set of commonly used sensors in past studies and other plausible ones as listed in the review articles [14], [15].

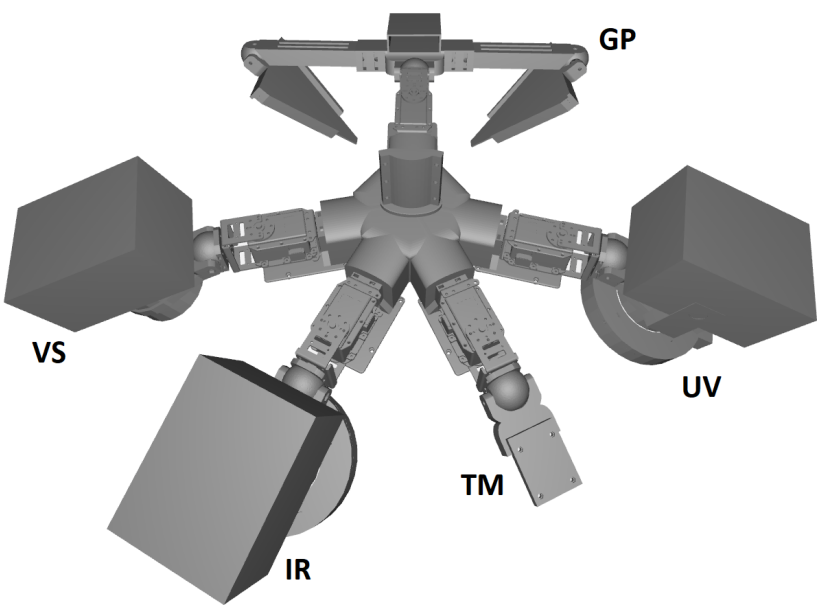

Fig. 1: The proposed Multi-Agent System (MAPS) and its sensors. More images are available in [13]

The software is divided among the agents of the system where every agent has a cooperative and distributed decisionmaking system $(\mathrm{CoD} 2 \mathrm{M})$, to make them capable of performing decisions individually. The DM processes which take place are 1) the next acquisition position for the sensor $(\alpha)$; 2) a decision whether or not a detected buried object is an IED by using the local information from the integrated sensor $(\beta)$; and collaboratively to decide if 3 ) a decision whether or not a detected buried object is an IED with the support received from the other agents $(\Omega)$.

It is important to highlight that the collaborative decision $(\Omega)$ can be considered as the definitive output of an agent as it plays the most important role in terms of IED detection. This is because once the five $\Omega$ values are available (i.e. one per agent), they are evaluated and the best one (e.g. the greatest) is the one marking the difference between an IED/non-IED classification (based on $\Omega$ being $<0.5$ or $\geq 0.5$ respectively).

In this study, several AI and machine-learning algorithms were chosen from the literature for implementing the three
DM processes. These algorithms are divided into three sets, intelligent, statistic aggregation and voting mode. Also, the tests made have all possible configurations among the selected decision-making methods, on each agent. This allowed making a comparative analysis in which a high number of DM systems were tested to recommend the most suitable distribution of training and validation samples to achieve the most robust decision-making system in terms of environmental conditions.

For the intelligent methods, we used an evolutionary methodology with two test Evolutionary Algorithms (EA). This was due to the possibility to encode individuals as neurons, activation functions, weighted connections, or even fuzzy rules [16], [17], to then manipulate them and generate new neural or fuzzy structures.

The first intelligent decision-making method (IDMM) applied for the $\alpha, \beta$ and $\Omega$ decisions is a feed-forward artificial neural networks (ffANN) constructed using a neuroevolution technique. In this work, to train and implement the networks we used the NeuroEvolution of Augmenting Topologies (NEAT) algorithm originally designed in [18]. This method is currently used for evolving problem-specific neural structures that would be difficult to devise otherwise [19]. It contains a GA customised to efficiently optimise parameters like the number of neurons, layers, activation function, connection weights, etc., of an artificial neural network according to the strategy explained in [18], [17]).

A second IDMM is a EA variant designed and used in this study to evolve a fuzzy decision support system (FDSS). We use a variant of the discrete GA in [16], whose individuals (i.e. candidate solutions) encode triangular membership functions. The proposed variant is almost identical to the one in [16] and employs the same two mutation operators as well as the same three basic crossover strategies, but differs from the original implementation as an additional fourth two-points crossover is used to add more diversity in the generated offspring solutions. This simple modification was tested empirically and adopted as it resulted in better performance. This second method is used for the $\alpha$ and $\Omega$ decisions.

For the case of the aggregation methods, it was only used to reach the $\Omega$ decision. There are three mathematical aggregations (AG) operators maximum (max), average (avg) and median (mdn). They are applied into a set B which contains the five $\beta$ values from each agent, to return:

$$
\lambda_{A G_{i}}=\left\{\begin{array}{lll}
\max (\mathrm{B}) & \text { if } & i=1 \\
\operatorname{avg}(\mathrm{B}) & \text { if } & i=2 \\
\operatorname{mdn}(\mathrm{B}) & \text { if } & i=3
\end{array} \quad i=1,2,3\right.
$$

And finally, for the last method, we tested the voting mode $\lambda_{\mathrm{VP}}$. This is an approach to aggregate all five $\beta$ values. An agent has a positive vote (i.e. has detected an IED) when its local decision value $\beta \geq 0.5$ and a quorum $\left(\lambda_{\mathrm{VP}}=1\right)$ is reached with at least three votes (since there are five agents in total), otherwise, $\lambda_{\mathrm{VP}}=0$. Similar to the previous case, this method is only used to reach the outcome given by $\Omega$ decision. 
Table I has a summary of the abbreviations of the decisionmaking methods evaluated and two methods which give the angle value for the next acquisition position $(\alpha)$ without an algorithmic process, a fixed point (FP) and a random value (RND). These abbreviations are useful to show the best results listed in figures 5 and 6

\begin{tabular}{|c||c|c|c|c|c|c|c|c|}
\hline \hline Method & ffANN & FDSS & FP & RND & $\lambda_{V P}$ & $\lambda_{A G_{1}}$ & $\lambda_{A G_{2}}$ & $\lambda_{A G_{3}}$ \\
\hline Abbreviation & $\mathrm{N}$ & $\mathrm{F}$ & $\mathrm{P}$ & $\mathrm{R}$ & $\mathrm{V}$ & $\mathrm{M}$ & $\overline{\mathrm{B}}$ & $\dot{\mathrm{B}}$ \\
\hline
\end{tabular}

TABLE I: Abbreviations list for the decision-making methods.

To use, test or benchmark against this system, all source code is available online in [20] as well as in the project's collection [13] along with the data sets.

\section{EXPERIMENTS AND BENCHMARKING}

We selected the required metrics to evaluate the performance of CoD2M-MAPS and its detection accuracy as explained below. It can be noted that almost in the totality of the cases $\Omega$ and $\beta$ are random values distributed normally in the $[0,1]$ range. However, these results can be divided into two sets, to binarize the results, employing a threshold value. Since, there are only two classes, IED and no-IED.

The most usual metrics for binary classifiers are derived from the confusion matrix [21], which can be used to annotate the occurrences of a True Positive (TP), a True Negative (TN), a False Positive (FP) and a False Negative (FN) outcome as shown in table II. This leads to the calculation of

1) the True Positive Rate (TPR):

$$
\mathrm{TPR}=\frac{\mathrm{TP}}{\mathrm{TP}+\mathrm{FN}}=\frac{\mathrm{TP}}{\mathrm{P}} ;
$$

2) the False Positive Rate (FPR):

$$
\mathrm{FPR}=\frac{\mathrm{FP}}{\mathrm{FP}+\mathrm{TN}}=\frac{\mathrm{FP}}{\mathrm{N}},
$$

3) and, the accuracy (ACC):

$$
\mathrm{ACC}=\frac{\mathrm{TP}+\mathrm{TN}}{\mathrm{P}+\mathrm{N}} .
$$

\begin{tabular}{|c|c|c|c|}
\hline & \multicolumn{2}{|c|}{$\begin{array}{c}\text { True } \\
\text { condition }\end{array}$} \\
\hline & & Negative & Positive \\
\hline \multirow{2}{*}{$\begin{array}{l}\text { Decision } \\
\text { selected }\end{array}$} & Negative & $\mathrm{TN}$ & FN \\
\hline & Positive & FP & TP \\
\hline
\end{tabular}

TABLE II: Confusion matrix. In this specific study, the term "positive" refers to the detection of an IED, while "negative" of a non-IED.

In addition, a Receiver Operating Characteristic (ROC) curve [21] can display FPR (usually reported on X-axis) and TPR (usually reported on the y-axis) per threshold value. A point in the ROC space is interpreted as a better classification than another one if it is closer to $(0,1)$, as it means that there are only TP and no FP occurrences. Any classifier with a ROC curve or values below the line $x=y$ displays worse performances than a decision made with a random guess. For this reason, the area under the ROC curve (AUC) evaluation metric is commonly used with ROC to compare between classifiers, as its value for a random guess is known and equal to 0.5. Thus, a classifier with $\mathrm{AUC} \leq 0.5$ should never be taken under consideration, while AUC values greater than 0.5 indicate suitable classifiers. In this light, the best $\beta$, and the best and worst $\Omega$ outcome decisions obtained were plotted in the ROC space in figures 5 and 6 , per each distribution sample case.

As a complement of these binary classification metrics is the Root Mean Square Error (RMSE), defined as

$$
\mathrm{RMSE}=\sqrt{\frac{1}{S} \sum_{i=1}^{S}\left(x_{i}-\widehat{x}_{i}\right)^{2}}
$$

with $x_{i}$ being the expected outcome, $\widehat{x_{i}}$ the predicted output and $\mathrm{S}$ the training data-set size. This metric is selected since it is normalised by the sample size $S$ thus allowing for a fair comparison between different DM methods.

\section{A. Experimental set-up}

The Cartesian robot visible in [13] was built to move CoD2M-MAPS across a test terrain of size is $670 \times 1100$ $\mathrm{mm}$ with a fixed scanning step of $50 \mathrm{~mm}$. To simulate a real scenario, three mock improvised explosive devices (IEDs) were buried at locations $(550,250),(350,600)$ and $(500,850)$. This test terrain was kept as intact a possible to avoid undesired debris, e.g. city garbage, to contaminate the tests. As in real improvised land mines, plastic bottles and PVC pipes were used for the body of the mock mine while syringes and wooden clothespins were used for simulating the detonation system. The land was left undisturbed time enough for vegetation to grow back and look even again.

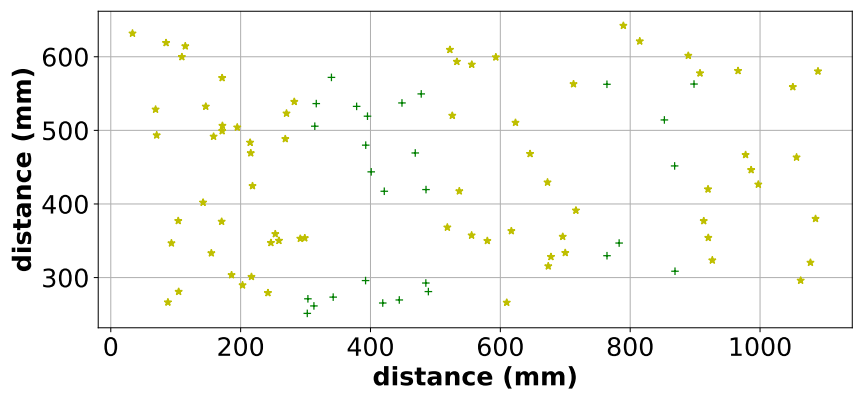

Fig. 2: Training (green crosses) and validation (yellow crosses) samples distribution.

We collected 100 samples at fixed positions for two consecutive days for both training and validation (positions are presented in figure 2). As expected in real life outdoor scenarios, each day was under different conditions of illumination and relative humidity. For day one (30) sensor acquisitions were made during the morning with a dry day. Samples from 


\begin{tabular}{|c|c|c|c|c|c|c|c|c|c|}
\cline { 2 - 11 } \multicolumn{1}{c|}{} & \multicolumn{4}{c|}{$C_{1}$} & \multicolumn{3}{c|}{$C_{2}$} & \multicolumn{3}{c|}{$C_{3}$} \\
\hline SEN & ACC & RMSE & AUC & ACC & RMSE & AUC & ACC & RMSE & AUC \\
\hline \hline VS & 0,690 & $\mathbf{0 , 4 6 5}$ & 0,775 & 0,640 & 0,488 & 0,826 & 0,678 & 0,544 & $\mathbf{0 , 9 0 6}$ \\
\hline IR & $\mathbf{0 , 7 3 0}$ & 0,498 & $\mathbf{0 , 8 4 6}$ & 0,600 & 0,572 & 0,771 & $\mathbf{0 , 7 8 5}$ & $\mathbf{0 , 4 1 1}$ & 0,818 \\
\hline UV & 0,660 & 0,480 & 0,743 & $\mathbf{0 , 8 2 0}$ & $\mathbf{0 , 3 7 3}$ & $\mathbf{0 , 8 9 1}$ & 0,535 & 0,549 & 0,853 \\
\hline TM & 0,630 & 0,527 & 0,527 & 0,490 & 0,533 & 0,535 & 0,464 & 0,524 & 0,742 \\
\hline GP & 0,340 & 0,504 & 0,500 & 0,590 & 0,453 & 0,676 & 0,785 & 0,418 & 0,830 \\
\hline
\end{tabular}

(a) Local decision-making $(\beta)$

\begin{tabular}{|c|c|c|c|c|c|c|c|c|c|}
\cline { 2 - 11 } \multicolumn{1}{c|}{} & \multicolumn{4}{c|}{$C_{1}$} & \multicolumn{3}{c|}{$C_{2}$} & \multicolumn{3}{c|}{$C_{3}$} \\
\hline SEN & ACC & RMSE & AUC & ACC & RMSE & AUC & ACC & RMSE & AUC \\
\hline \hline VS & 0,780 & 0,394 & 0,812 & 0,690 & 0,416 & 0,855 & 0,678 & 0,477 & 0,912 \\
\hline IR & 0,740 & $\mathbf{0 , 3 8 3}$ & 0,868 & 0,690 & 0,419 & 0,849 & 0,678 & 0,494 & 0,760 \\
\hline UV & 0,760 & 0,428 & 0,803 & 0,770 & 0,447 & 0,685 & 0,571 & 0,539 & 0,947 \\
\hline TM & $\mathbf{0 , 8 1 0}$ & 0,416 & 0,765 & $\mathbf{0 , 8 5 0}$ & $\mathbf{0 , 3 3 1}$ & $\mathbf{0 , 9 0 1}$ & $\mathbf{0 , 7 8 5}$ & 0,454 & 0,631 \\
\hline GP & 0,740 & 0,412 & $\mathbf{0 , 8 7 6}$ & 0,680 & 0,442 & 0,862 & 0,678 & $\mathbf{0 , 3 9 8}$ & $\mathbf{1 , 0 0 0}$ \\
\hline
\end{tabular}

(b) Cooperative decision-making $(\Omega)$

TABLE III: Metrics of the best accuracy result per sensor and per case for the training data set

day two (31) were during the afternoon and were under drizzle conditions.

Moreover, an assessment of the decision-making system performance according to the distribution of the samples was made for three cases. The first case $\left(C_{1}\right)$ uses the samples from day one as the training data set and the models obtained are validated with the samples from day two. For the second case $\left(C_{2}\right)$, the samples from day two are used for the training set and the samples from day one for the validation stage. And finally, the third case $\left(C_{3}\right)$ divides the samples of both days into two regions indicated in figure 2 by the colour of the crosses. These regions guaranteed that both sets included data with IED and non-IED samples.

\section{B. Performance evaluation}

To execute an evaluation of the decision-making systems obtained, the results of the training and validation stages are compared per each case.

Tables IIIa and IIIb belong to the results of the training stage, and tables IVa and IVb to the validation stage, local and cooperative models respectively. Each table presents the best accuracy model per agent and per case. Also, these tables show the results of each model according to three metrics: the accuracy (ACC - see equation 4), the root mean square error (RMSE - see equation 5), and the AUC. Besides, the best result per column (per metric) are shown in black bold text.

A contrast between tables [II and IV show us some behaviours. First, the best accuracy value among training and validation stages decrements for cases 1 and 2 , in contrast, results in case 3 have a similar performance. Second, the best RMSE value in the validation results (see table IV) belong to case 3 , in both cases, local and cooperative model decisions. Although, the best performance for the RMSE metric in the training case belongs to case 2 . And third, the highest AUC values per table, it means the best results of the AUC metric, are related to case 3 . This last observation is also discernible

\begin{tabular}{|c|c|c|c|c|c|c|c|c|c|}
\cline { 2 - 11 } \multicolumn{1}{c|}{} & \multicolumn{4}{c|}{$C_{1}$} & \multicolumn{3}{c|}{$C_{2}$} & \multicolumn{3}{c|}{$C_{3}$} \\
\hline SEN & ACC & RMSE & AUC & ACC & RMSE & AUC & ACC & RMSE & AUC \\
\hline \hline VS & 0,660 & 0,583 & 0,220 & 0,670 & 0,572 & 0,528 & $\mathbf{0 , 7 6 3}$ & 0,477 & 0,777 \\
\hline IR & $\mathbf{0 , 6 6 0}$ & 0,582 & 0,783 & 0,340 & 0,808 & $\mathbf{0 , 8 4 2}$ & 0,722 & $\mathbf{0 , 4 3 5}$ & 0,809 \\
\hline UV & 0,660 & 0,583 & $\mathbf{0 , 8 2 5}$ & 0,380 & 0,704 & 0,777 & 0,708 & 0,448 & $\mathbf{0 , 8 6 6}$ \\
\hline TM & 0,340 & 0,537 & 0,510 & $\mathbf{0 , 6 7 0}$ & $\mathbf{0 , 4 6 9}$ & 0,512 & 0,375 & 0,560 & 0,499 \\
\hline GP & 0,420 & $\mathbf{0 , 4 9 4}$ & 0,566 & 0,390 & 0,512 & 0,672 & 0,458 & 0,550 & 0,552 \\
\hline
\end{tabular}

(a) Local decision-making $(\beta)$

\begin{tabular}{|c|c|c|c|c|c|c|c|c|c|}
\cline { 2 - 11 } \multicolumn{1}{c|}{} & \multicolumn{4}{c|}{$C_{1}$} & \multicolumn{3}{c|}{$C_{2}$} & \multicolumn{3}{c|}{$C_{3}$} \\
\hline SEN & ACC & RMSE & AUC & ACC & RMSE & AUC & ACC & RMSE & AUC \\
\hline \hline VS & 0,660 & 0,499 & 0,522 & 0,670 & 0,491 & 0,500 & 0,639 & 0,463 & 0,757 \\
\hline IR & 0,660 & 0,529 & 0,524 & 0,470 & 0,485 & $\mathbf{0 , 7 1 6}$ & $\mathbf{0 , 7 7 7}$ & $\mathbf{0 , 4 2 7}$ & 0,809 \\
\hline UV & $\mathbf{0 , 6 9 0}$ & $\mathbf{0 , 4 8 8}$ & 0,759 & 0,670 & 0,490 & 0,500 & 0,625 & 0,437 & 0,815 \\
\hline TM & 0,660 & 0,490 & $\mathbf{0 , 8 0 4}$ & 0,670 & 0,477 & 0,500 & 0,736 & 0,513 & 0,678 \\
\hline GP & 0,660 & 0,547 & 0,780 & $\mathbf{0 , 6 7 0}$ & $\mathbf{0 , 4 6 3}$ & 0,610 & 0,638 & 0,422 & $\mathbf{0 , 8 2 4}$ \\
\hline
\end{tabular}

(b) Cooperative decision-making $(\Omega)$

TABLE IV: Metrics of the best accuracy result per sensor and per case for the validation data set

in figure 4, where the mean values (orange line) of box-plot of the AUC results for case 3, are higher than the mean values from cases 1 and 2.

As a complement, figures 5 and 6 have the ROC curve per sensor and per case, local and cooperative, of the best results presented in the tables [II] and IV] in blue the local decision $(\beta)$, and green the cooperative decision model $(\Omega)$. These figures present the ROC curves per agent (each row) and per case (each column) where the resulting ROC curves are put together with the random result (yellow line) and the worst cooperative decision model per case (red line). Each cell has a box dialogue with the information related to the decision-making models drawn.

On the other hand, figures 3 and 4 have the box-plots of all the AUC results of the models evaluated, local $(\beta)$ and cooperative $(\Omega)$, respectively. In these figures, the columns are associated with the cases and the rows to the stages of model generation and evaluation (i.e. the first row shows the training stage while the second one the validation stage).

Figure 3 shows us that the information from the agent with a VS camera is the most affected by the distribution of the samples among training and validation. Since validation results of case one (figure 3d), show a performance decreasing for the AUC metric, in contrast to the performance of the same case for the training stage (figure 3a. Likewise, figure 6a presents the low performance with the green curve which is close to the random case (yellow line).

Similar behaviour is detected for case two, however, the performance for the validation results in case two seems to have a lower affectation (figure $3 \mathrm{e}$ and figure 6b. Additionally, the results of case 3 indicate that the agent with the VS sensor keeps the performance of the AUC metric for both stages, training (figure $3 \mathrm{c}$ ) and validation (figure $3 \mathrm{f}$ ). It implies that this model is most robust to the illumination affectation, in contrast to cases 1 and 2 . 


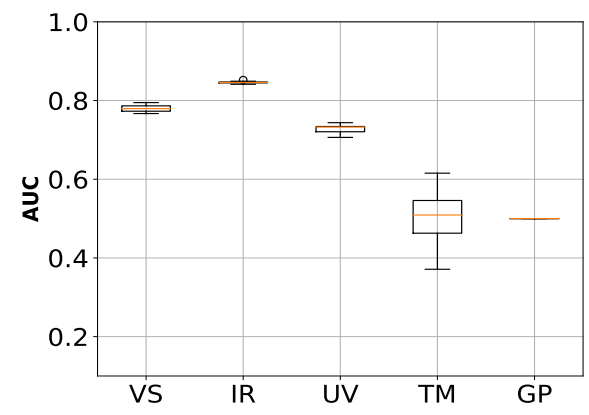

(a) Case 1. Training results

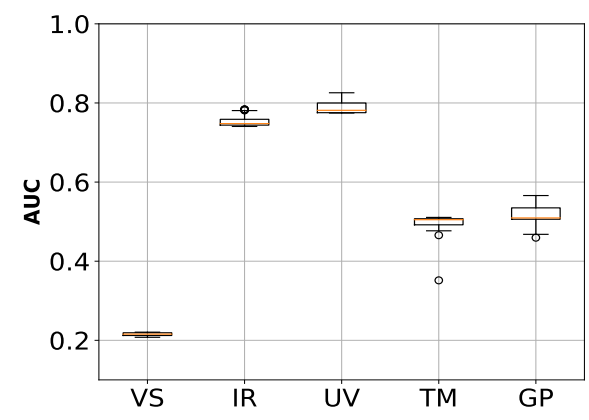

(d) Case 1. Validation results

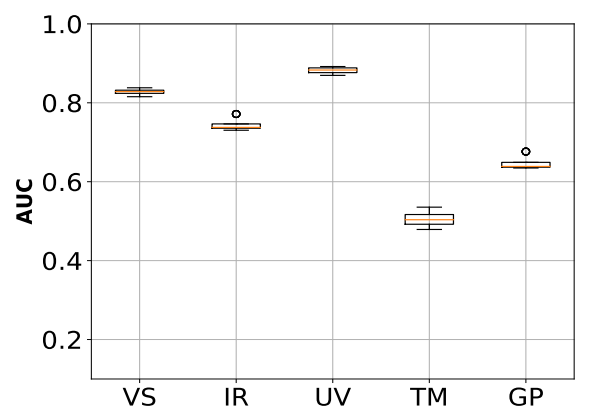

(b) Case 2. Training results

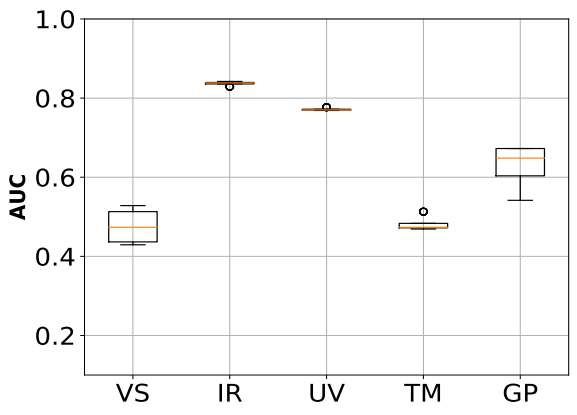

(e) Case 2. Validation results

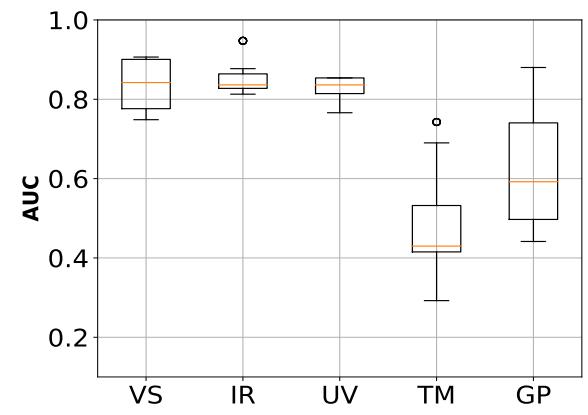

(c) Case 3. Training results

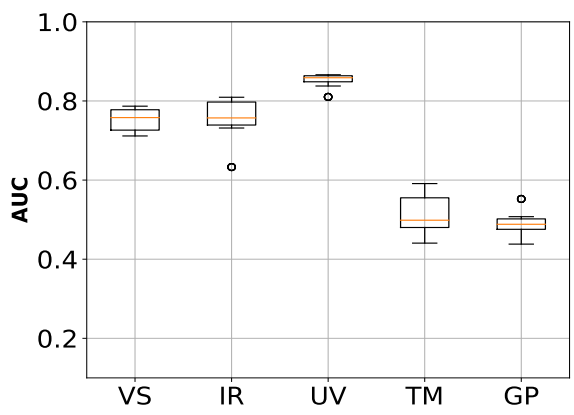

(f) Case 3. Validation results

Fig. 3: Distribution of the AUC for the local decision models per type of sensor and per stage.

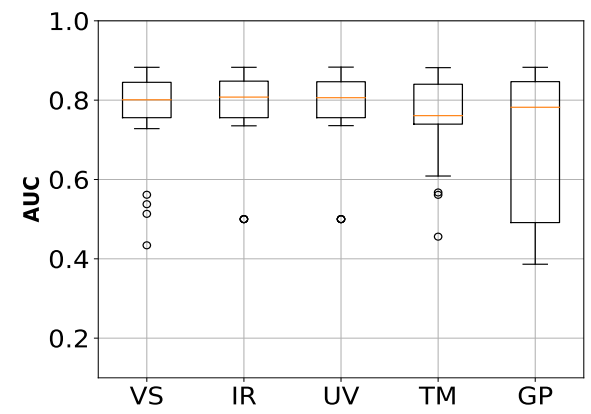

(a) Case 1. Training results

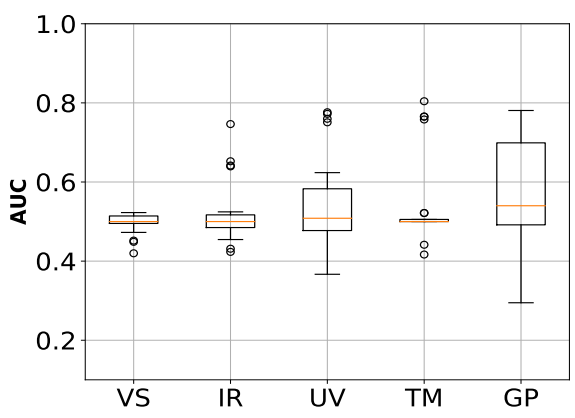

(d) Case 1. Validation results

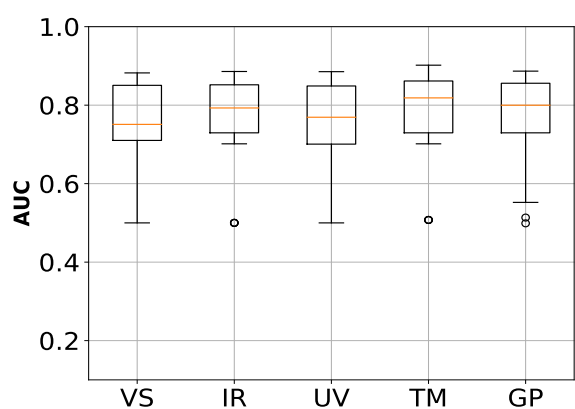

(b) Case 2. Training results

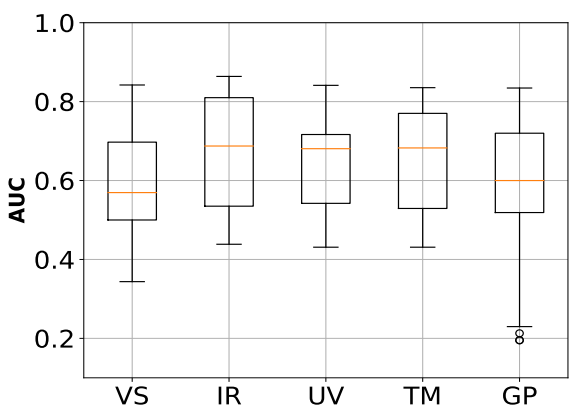

(e) Case 2. Validation results

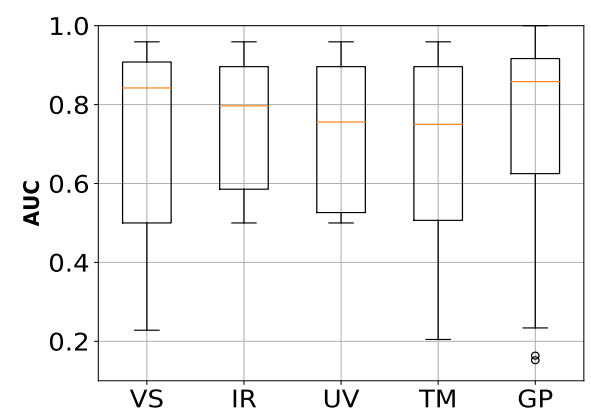

(c) Case 3. Training results

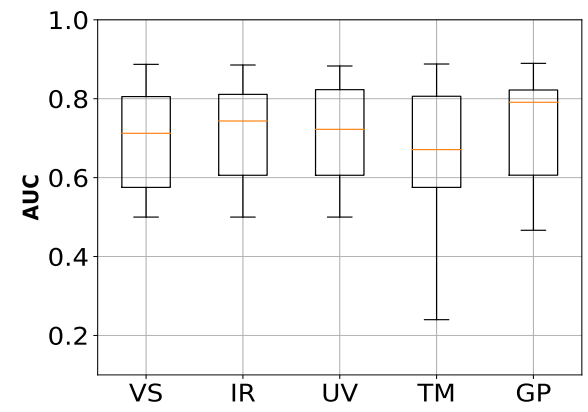

(f) Case 3. Validation results

Fig. 4: Distribution of the AUC for the cooperative decision models per type of sensor and per stage. 


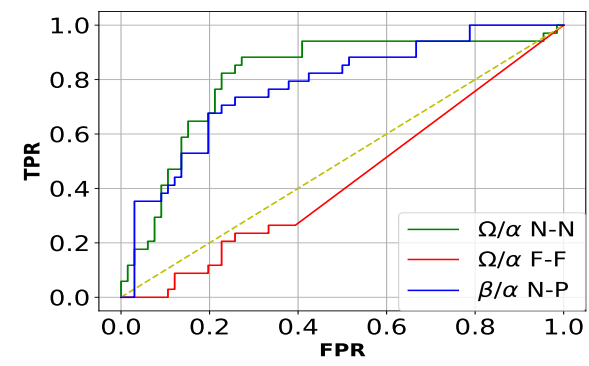

(a) VS - case 1

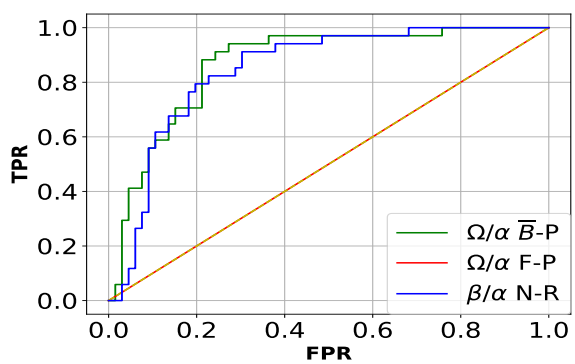

(d) IR - case 1

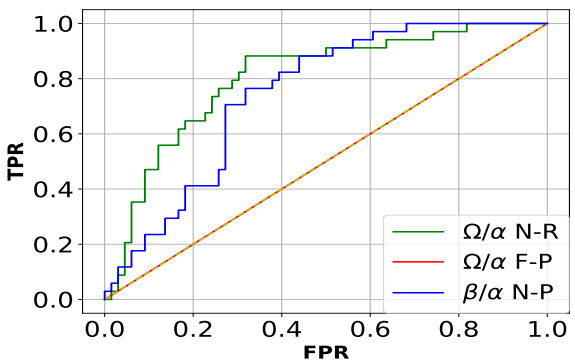

(g) UV - case 1

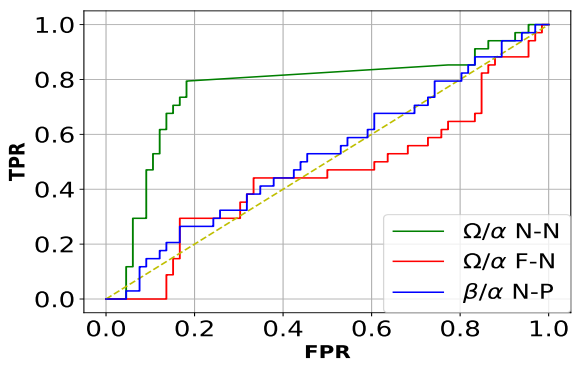

(j) $\mathrm{TM}$ - case 1

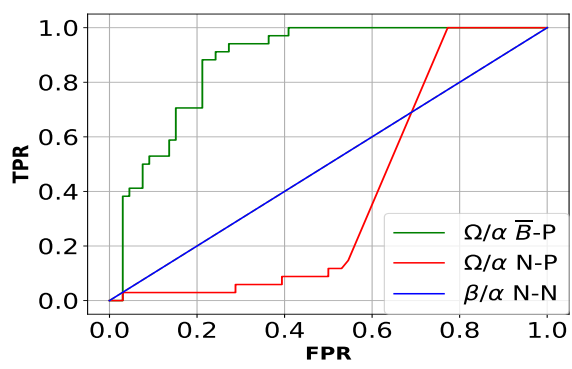

(m) GP - case 1

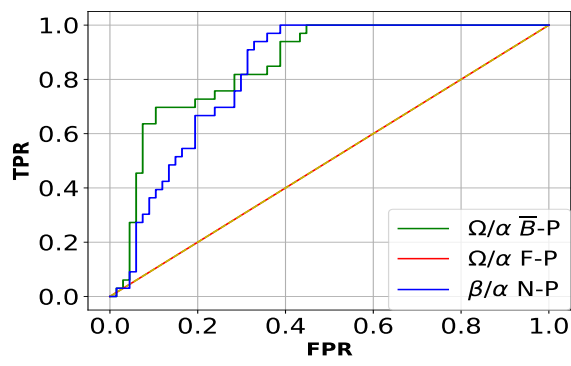

(b) VS - case 2

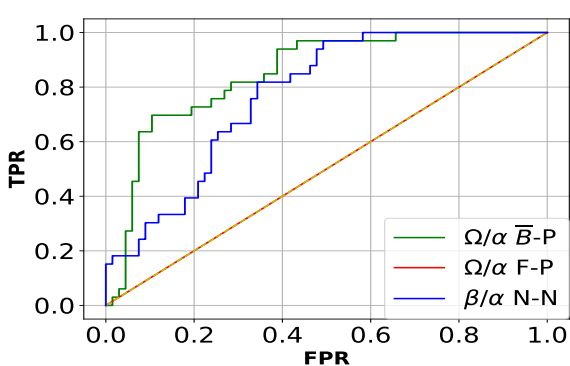

(e) IR - case 2

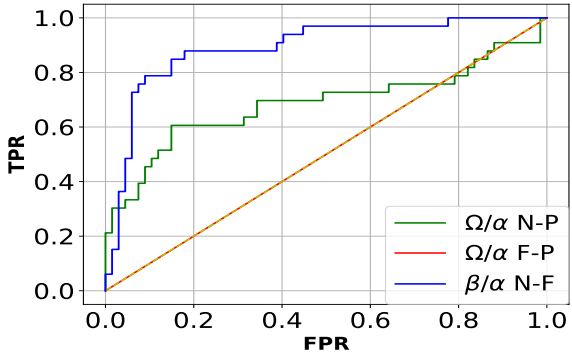

(h) UV - case 2

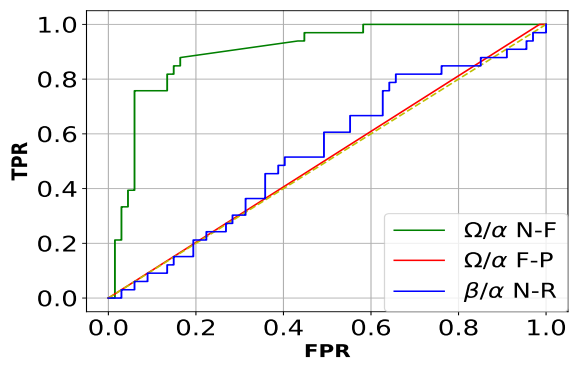

(k) TM - case 2

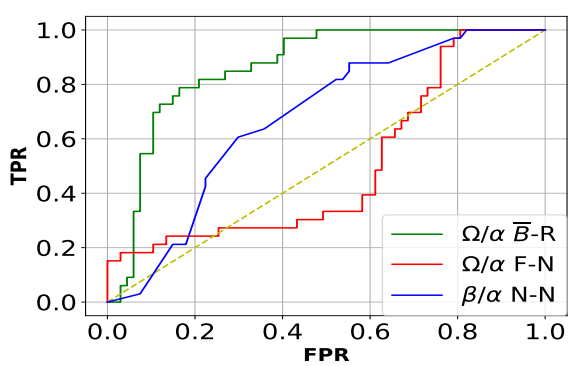

(n) GP - case 2

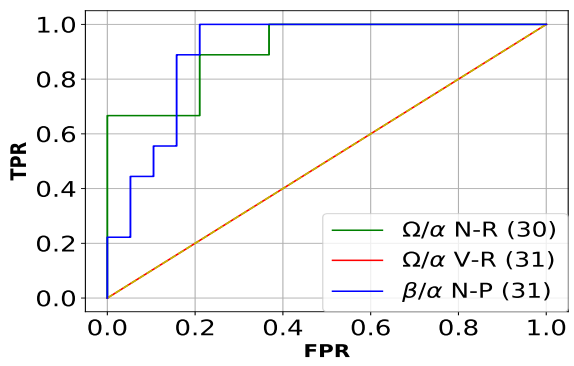

(c) VS - case 3

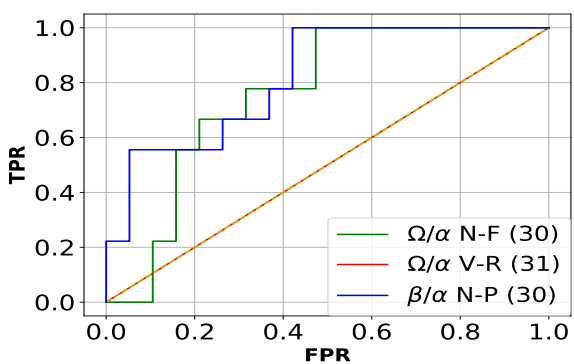

(f) IR - case 3

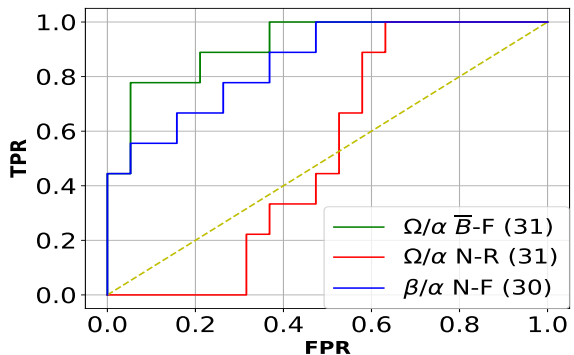

(i) UV - case 3

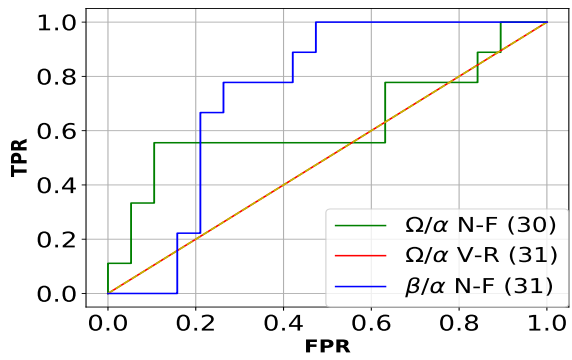

(1) $\mathrm{TM}$ - case 3

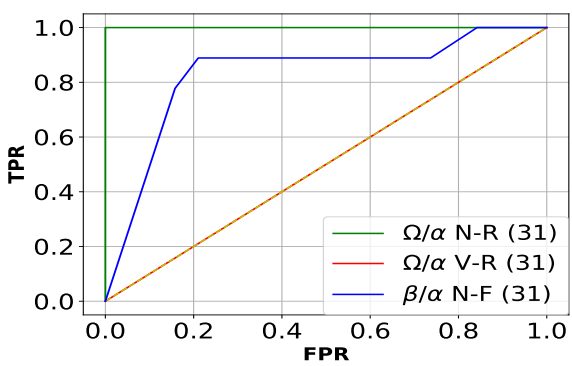

(o) GP - case 3

Fig. 5: Selected ROC curves from training data set per sensor and per case. Green curve belongs to the best ACC result of the cooperative decision-making, the red one to the worst cooperative result, and the blue one to the best local decision-making result. 


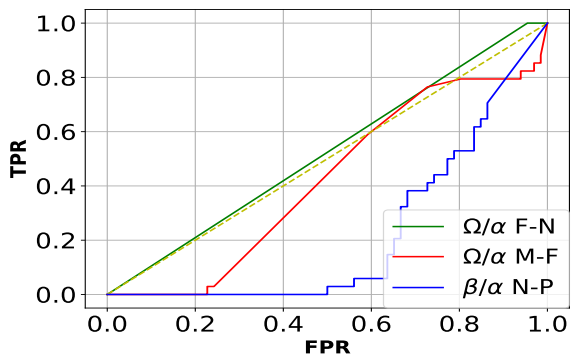

(a) VS - case 1

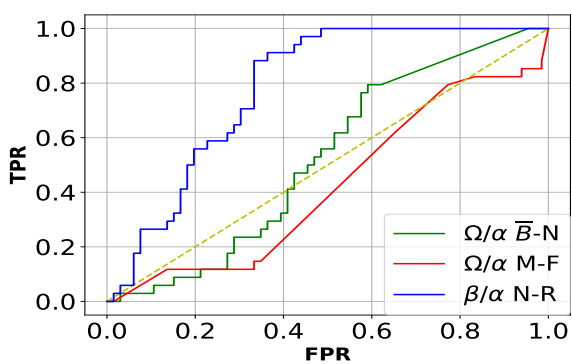

(d) IR - case 1

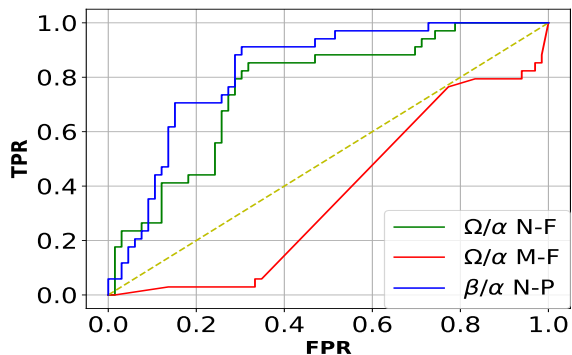

(g) UV - case 1

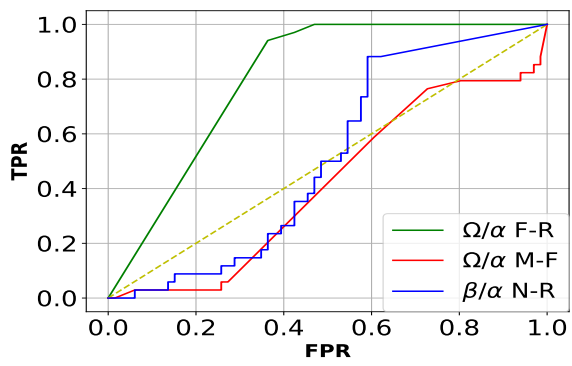

(j) $\mathrm{TM}$ - case 1

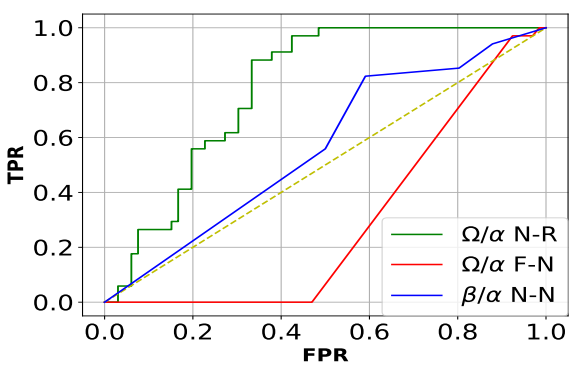

(m) GP - case 1

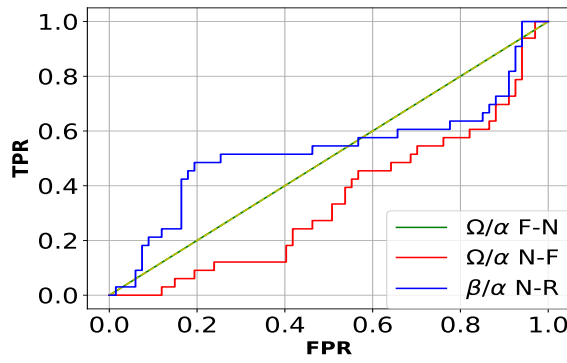

(b) VS - case 2

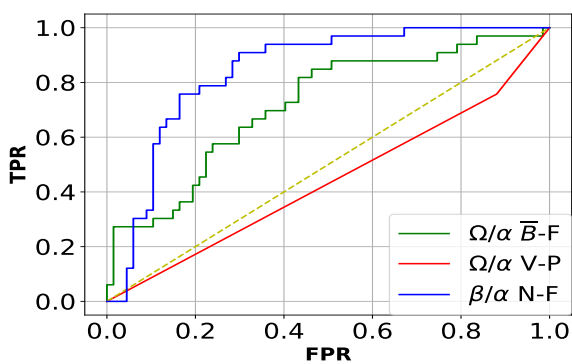

(e) IR - case 2

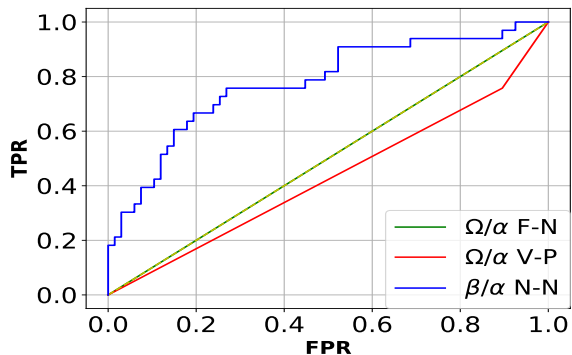

(h) UV - case 2

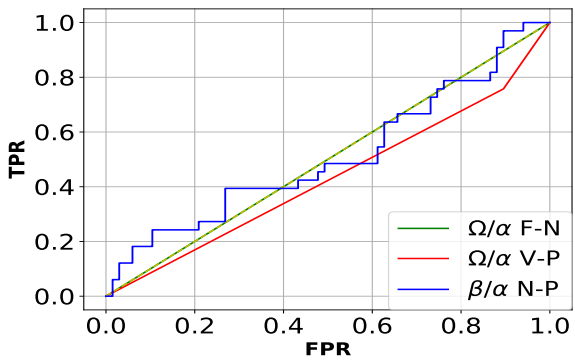

(k) TM - case 2

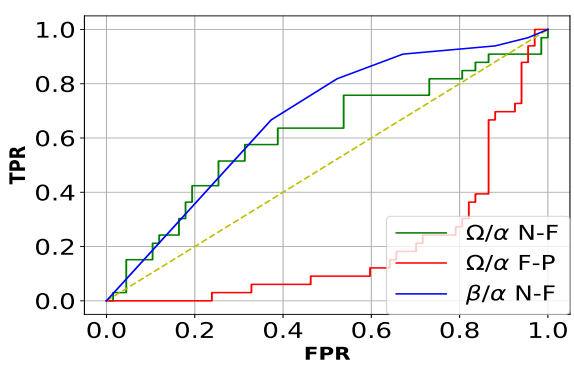

(n) GP - case 2

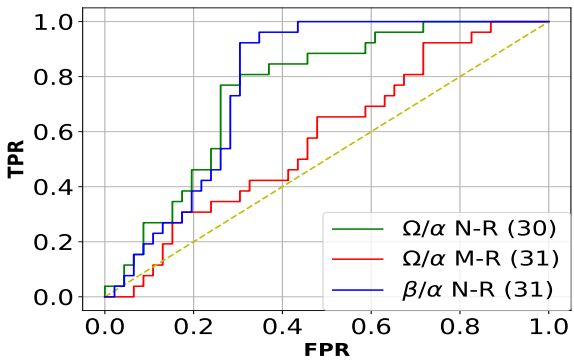

(c) VS - case 3

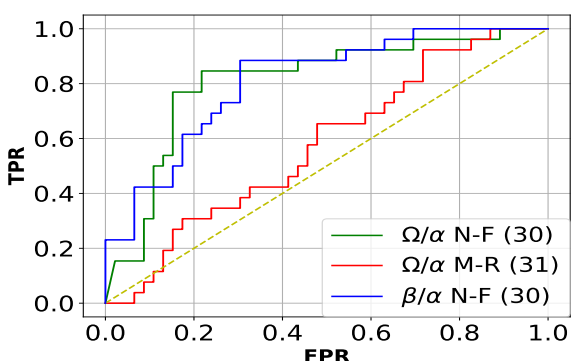

(f) IR - case 3

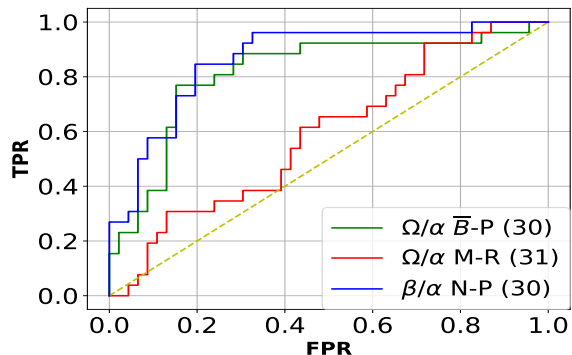

(i) UV - case 3

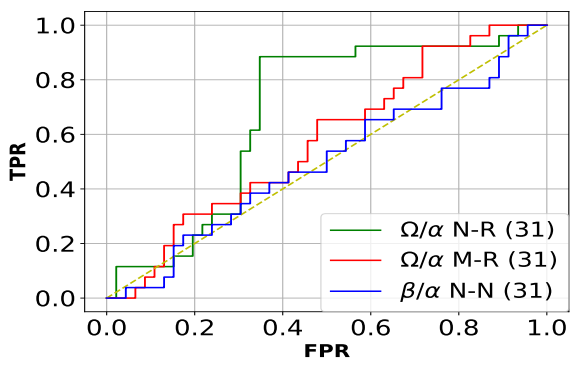

(1) $\mathrm{TM}$-case 3

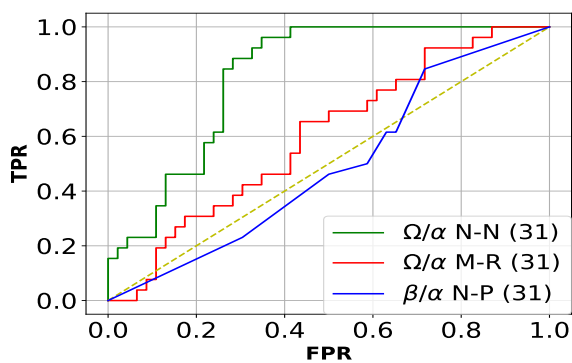

(o) GP - case 3

Fig. 6: Selected ROC curves from validation data set per sensor and per case. Green curve belongs to the best ACC result of the cooperative decision-making, the red one to the worst cooperative result, and the blue one to the best local decision-making result. 
Moreover, figure 4 shows that the cooperative decision process increases and balance the AUC metric per agent since the integration of the local decisions contributes to the reduction of the uncertainty of the global decision. For the training stage, cases 1 and 2 have a similar distribution and mean value (orange line), the opposite happens for case three where the results are more scattered; the best results belong to case 3. A different result distribution can be seen at the validation stage, in which case 1 has the worst AUC values (figure 4d). Moreover, the AUC values distribution among cases 2 and 3 are similar. Nonetheless, the best achievement and mean values are given by case 3 (figures $4 \mathrm{c}$ and $4 \mathrm{f}$ ). This case is the only one that has consistent performances of the AUC metric across data sets.

\section{Conclusion and Future Direction}

Land mine detection is a task with many external constraints and challenges. This means that an effective detection model needs to avoid or reduce the sensor noise to achieve a robust decision. Our study of IEDs detection resulted in a system to integrate different sources of information; this study evaluates the performance of the decision-making models according to the training and validation set.

The distribution methodology of samples from the data set into training and validation is crucial when these samples are taken under the influence of varied external conditions; in this case, is particularly critical.

Looking at 3 cases considered, even though the performance in the training stage of the cases 1 and 2 are better than case 3 , it is the validation stage determines the real performance of the models when they are tested with fresh data. The information recorded in the tables and figures in section IV-B indicates that samples distribution used for case 3 result in the best performance consistency between the training and validation stage. It means that the intelligent decision-making models from samples altered by different conditions are less sensible to sensor noise produced by external parameters.

An extension of this work is to acquire more samples with our CoD2M-MAPS with other environmental conditions (humidity and sunlight), terrains (sand and clay) and vegetation; to consider IEDs as disseminated along to the world. With these new samples, we can perform a new evaluation of the distribution of the samples to obtain the most robust decisionmaking system. On the other hand, the extension of the data set will allow evaluating and contrasting future IEDs detection systems.

\section{ACKNOWLEDGEMENT}

The "Collaborative methodology for enhancing sustainability in rural communities and the use of land" project [22] received funds from De Montfort University (Leicester,UK), Colciencias (Colombia, [grant number 647, 2014]) and Pontificia Universidad Javeriana (Bogotá, Colombia, [grant number VRI-05,2017]).

\section{REFERENCES}

[1] M. K. Habib, "Humanitarian Demining : Reality and the Challenge of Technology - The State of the Arts," International Journal of Advanced Robotic Systems, vol. 4, no. 2, pp. 151-172, 2007.

[2] P. C. Bhope and A. S. Bhalchandra, "Various landmine detection techniques: A review," International Journal of Innovative Research in Science, Engineering and Technology, vol. 4, no. 6, pp. 771-775, 2015.

[3] K. Takahashi, H. Preetz, and J. Igel, "Soil properties and performance of landmine detection by metal detector and ground-penetrating radar - Soil characterisation and its verification by a field test," Journal of Applied Geophysics, vol. 73, no. 4, pp. 368-377, 2011.

[4] J. Prado and L. Marques, "Reducing false-positives in multi-sensor dataset of landmines via sensor fusion regularization," in 2017 IEEE International Conference on Autonomous Robot Systems and Competitions, ICARSC 2017, 2017, pp. 204-209.

[5] P. A. Prada and M. C. Rodriguez, "Demining Dogs in Colombia - A Review of Operational Challenges, Chemical Perspectives, and Practical Implications," Science and Justice, vol. 56, no. 4, pp. 269-277, 2016.

[6] L. E. Besaw and P. J. Stimac, "Deep convolutional neural networks for classifying gpr b-scans," in Detection and Sensing of Mines, Explosive Objects, and Obscured Targets XX, vol. 9454. International Society for Optics and Photonics, 2015, p. 945413.

[7] J. Colorado, C. Devia, M. Perez, I. Mondragon, D. Mendez, and C. Parra, "Low-altitude autonomous drone navigation for landmine detection purposes," in 2017 International Conference on Unmanned Aircraft Systems (ICUAS). IEEE, June 2017, pp. 540-546.

[8] T. Dogaru, B. Phelan, and D. Liao, "Imaging of buried targets using UAV-based, ground penetrating, synthetic aperture radar," in Radar Sensor Technology XXIII, K. I. Ranney and A. Doerry, Eds., vol. 11003, International Society for Optics and Photonics. SPIE, 2019, pp. 18-35. [Online]. Available: https://doi.org/10.1117/12.2519116

[9] G. Mathews and H. Durrant-Whyte, "Decentralised Decision Making for Ad-hoc Multi-Agent Systems," in Advances in Applied Self-Organizing Systems, M. Prokopenko, Ed. London: Springer-Verlag, 2013, ch. 5, pp. $91-133$.

[10] D. Ye, M. Zhang, and A. V. Vasilakos, "A Survey of Self-Organization Mechanisms in Multiagent Systems," IEEE Transactions on Systems, Man, and Cybernetics: Systems, vol. 47, no. 3, pp. 441-461, mar 2017.

[11] Z. Zuo, Q. Han, B. Ning, X. Ge, and X. Zhang, "An overview of recent advances in fixed-time cooperative control of multiagent systems," IEEE Transactions on Industrial Informatics, vol. 14, no. 6, pp. 2322-2334, June 2018.

[12] H. Liang, Y. Zhang, T. Huang, and H. Ma, "Prescribed performance cooperative control for multiagent systems with input quantization," IEEE Transactions on Cybernetics, pp. 1-10, 2019.

[13] J. Florez-Lozano, F. Caraffini, M. Gongora, and C. Parra, "Multi-agent robotic system dataset," jun 2019. [Online]. Available: https://dmu.figshare.com/collections/Multi-agent $\left\{\_\right\}$ robotic $\left\{\begin{array}{l}\text { _ } \\ \text { system }\{\text { _ }\} \text { dataset } / 4555424 / 2\end{array}\right.$

[14] L. Robledo, M. Carrasco, and D.Mery, "A survey of land mine detection technology," International Journal of Remote Sensing, vol. 30, no. 9, pp. 2399-2410, 2009.

[15] J. Florez and C. Parra, "Review of sensors used in robotics for humanitarian demining application," in 2016 IEEE Colombian Conference on Robotics and Automation (CCRA). Bogota: IEEE, sep 2016, pp. 1-6.

[16] L. Baron, S. Achiche, and M. Balazinski, "Fuzzy decision support system knowledge base generation using a genetic algorithm," International Journal of Approximate Reasoning, vol. 28, no. 2-3, pp. 125-148, 2001.

[17] K. O. Stanley, J. Clune, J. Lehman, and R. Miikkulainen, "Designing neural networks through neuroevolution," Nature Machine Intelligence, vol. 1, no. 1, pp. 24-35, 2019

[18] K. O. Stanley, "Efficient Evolution of Neural Networks through Complexification," Ph.D. dissertation, University of Texas at Austin, 2004.

[19] A. Soltoggio, K. O. Stanley, and S. Risi, "Born to learn: The inspiration, progress, and future of evolved plastic artificial neural networks," Neural Networks, vol. 108, pp. $48-67,2018$.

[20] J. Florez-Lozano, F. Caraffini, M. Gongora, and C. Parra, "Cod2m-maps source code," https://doi.org/10.21253/DMU.9630779.v1 Aug 2019.

[21] T. Fawcett, "An introduction to roc analysis," Pattern Recognition Letters, vol. 27, no. 8, pp. 861-874, jun 2006.

[22] F. Caraffini and M. Gongora, "Collaborative methodology for enhancing sustainability in rural communities and the use of land," https://doi.org/ 10.21253/DMU.8483960 2019 